\title{
Adsorption-induced symmetry reduction of metal-phthalocyanines studied by vibrational spectroscopy
}

\author{
J. Sforzini, F. C. Bocquet, ${ }^{*}$ and F. S. Tautz \\ Peter Grünberg Institut (PGI-3), Forschungszentrum Jülich, 52425 Jülich, Germany \\ and Jülich Aachen Research Alliance (JARA), Fundamentals of Future Information Technology, 52425 Jülich, Germany \\ (Received 25 September 2016; revised manuscript received 14 August 2017; published 5 October 2017)
}

\begin{abstract}
We investigate the vibrational properties of Pt- and Pd-phthalocyanine (PtPc and PdPc) molecules on $\mathrm{Ag}(111)$ with high-resolution electron energy loss spectroscopy (HREELS). In the monolayer regime, both molecules exhibit long-range order. The vibrational spectra prove a flat adsorption geometry. The redshift of specific vibrational modes suggests a moderate interaction of the molecules with the substrate. The presence of asymmetric vibrational peaks indicates an interfacial dynamical charge transfer (IDCT). The molecular orbital that is involved in IDCT is the former $E_{\mathrm{g}}$ lowest unoccupied molecular orbital (LUMO) of the molecules that becomes partially occupied upon adsorption. A group-theoretical analysis of the IDCT modes, based on calculated vibrational frequencies and line shape fits, provides proof for the reduction of the symmetry of the molecule-substrate complex from fourfold $D_{4 h}$ to $C_{2 v}\left(\sigma_{v}\right), C_{s}\left(\sigma_{v}\right)$, or $C_{2}$ and the ensuing lifting of the degeneracy of the former LUMO of the molecule. The vibration-based analysis of orbital degeneracies, as carried out here for $P \operatorname{tPc} / \operatorname{Ag}(111)$ and $\mathrm{PdPc} / \mathrm{Ag}(111)$, is particularly useful whenever the presence of multiple molecular in-plane orientations at the interface makes the analysis of orbital degeneracies with angle-resolved photoemission spectroscopy difficult.
\end{abstract}

DOI: 10.1103/PhysRevB.96.165410

\section{INTRODUCTION}

Organic molecules with $\pi$-conjugated electron systems have been intensively studied in recent years. Apart from a fundamental interest in their electronic properties, this activity is motivated by the wide range of possible applications in the fields of optoelectronics [1,2] and spintronics [3]. Among these molecules, metal-phthalocyanines (MPc), i.e., tetrabenzoporphyrazine macrocycles with a metal atom in their center, play an important role, because of their planar geometry, their thermal stability, their suitability for organic molecular beam epitaxy, and their chemical versatility that its brought about by very diverse central metal atoms [4]. In fact, MPc molecules have been employed in organic light-emitting diodes [5], field effect transistors [6,7], and solar cells [8].

The interaction of MPc with metal surfaces is interesting both from a fundamental point of view, in particular regarding the balance between the contributions of the central metal atom and the $\pi$-electron system, and for applications, in which such interfaces are the primary functional elements. For MPc with almost filled $d$ shells, the molecule-substrate interaction is dominated by the chemisorptive or physisorptive interaction of the macrocycle with the metal surface [4]. If there is a chemisorptive contribution to this bond, this often leads to charge transfer from the metal into the lowest unoccupied molecular orbital (LUMO) of the molecule [4]. This is also true in the case of PtPc and PdPc on $\mathrm{Ag}(111)$, as we show in this paper.

An interesting issue in relation to the molecule-substrate interaction in general and the charge transfer in particular is the

\footnotetext{
*f.bocquet@fz-juelich.de

Published by the American Physical Society under the terms of the Creative Commons Attribution 3.0 License. Further distribution of this work must maintain attribution to the author(s) and the published article's title, journal citation, and DOI.
}

symmetry of adsorbed MPc molecules. Except for a few cases, e.g., SnPc [9], the central metal fits into the inner cavity and the corresponding isolated MPc belongs to the $D_{4 h}$ point group [4]. Because of the presence of the surface, the symmetry of the molecule is lowered to $C_{4 v}$. However, a further molecular symmetry reduction to a twofold symmetry is often observed in scanning tunneling microscopy (STM) [9-14].

The symmetry reduction of MPc molecules from fourfold to twofold symmetry raises an intriguing question regarding the charge transfer into the molecule. The LUMO of isolated MPc is twofold degenerate and belongs to the irreducible representation $E_{\mathrm{g}}$ of the $D_{4 h}$ point group. This degeneracy is closely related to cross-conjugation in the central porphyrazine macrocycle of phthalocyanine molecules $[4,15]$. When charge is transferred into the LUMO, two situations are conceivable: either both orbitals remain degenerate and receive the same amount of charge, or the degeneracy is lifted and charge is transferred preferentially into one of the two. Degeneracy lifting has been invoked in certain cases to explain the symmetry reduction of MPc molecules $[11,14,16]$, while in other cases equal filling of both $E_{\mathrm{g}}$ orbitals was conjectured [17], or the symmetry reduction was assigned to structural effects $[10,13,18]$.

At first glance, STM seems to be the method of choice for symmetry analysis of molecular adsorbates, because any symmetry breaking should become immediately obvious in the image. However, in STM purely electronic as well as purely geometric effects can both lead to a symmetry reduction in the image, and there are only a few cases in which the origin of the broken symmetry can be determined on basis of STM alone (e.g., Ref. [16]). Of course, in most cases geometric and electronic effects will be coupled to each other, because a geometric distortion of the molecule by the influence of the external environment will also break the electronic symmetry within the molecule, and vice versa an electronic symmetry reduction will generally lead to a structural distortion (JahnTeller effect $[16,19,20])$. Yet, it is still an important question 
to ask which is the dominant of the two effects. For example, a strong geometric distortion may lead to a negligible symmetry breaking of electronic states.

For this reason, additional experimental methods have been employed to settle the issue of a possible lifting of the degeneracy. These include core level spectroscopy [14], near-edge x-ray absorption spectroscopy (NEXAFS) [21], or density functional theory (DFT) [14]. Recently, also photoemission tomography [22,23] has been applied to this problem: In the case of FePc/Ag(111) no splitting of the LUMO was found [17], while for $\mathrm{CuPc} / \mathrm{Ag}(110)$ a clear splitting was observed [24]. Photoemission tomography has the unique advantage that if a degeneracy lifting takes place, the actual orientation of the filled orbital can be determined [24]. However, this orientational sensitivity can also be a drawback, if many molecules with many different orientations are present at the surface. In this case, photoemission tomography results become difficult to analyze [17].

Here we present an approach that does not suffer from the presence of multiple orientations, because only the internal symmetry of the molecule-surface complex matters. Yet at the same time, if applicable, it leads to unambiguous results regarding electronic symmetry breaking, i.e., degeneracy lifting. This approach is based on vibrational spectroscopy. Specifically, we employ high-resolution electron energy loss spectroscopy (HREELS) which in its dipole scattering mechanism is sensitive to infrared (IR) active vibrational modes [25]. Due to the surface selection rule, only totally symmetric modes of the molecule-surface complex are IR active [25]. Any reduction of the molecular point group upon adsorption may imply that formerly inactive modes can become IR active. Because, as mentioned above, the symmetry reduction can be effected both by geometric and electronic effects, also the corresponding IR activation can have these two distinct origins. However, if additionally the line shape of an activated mode is taken into account, it is (under favorable conditions) possible to unambiguously link the activation of certain vibrational modes to an electronic symmetry breaking. More specifically, we argue here for the example of MPc molecules that if a mode that indicates a particular symmetry reduction has a Fano line shape, this proves an electronic contribution (i.e., degeneracy lifting) to this symmetry reduction, because the Fano line shape indicates an interfacial dynamical charge transfer (IDCT), and for an IDCT to be observable in a MPc molecule, there must exist an imbalance in the occupation of the two $E_{\mathrm{g}}$ LUMOs. Hence, their degeneracy must be broken.

\section{EXPERIMENTAL DETAILS}

The experiments were performed in an ultrahigh vacuum (UHV) system consisting of a preparation and an analysis chamber equipped with low energy electron diffraction (LEED) and HREELS. The pressures in the chambers were $4 \times 10^{-9}$ and $4 \times 10^{-10} \mathrm{mbar}$, respectively. The $\operatorname{Ag}(111)$ crystal surface was prepared by $\mathrm{Ar}^{+}$sputtering at $1 \mathrm{keV}$ followed by annealing to $730 \mathrm{~K}$ until a sharp LEED pattern was observed. Thin films of PdPc and PtPc were prepared in UHV by depositing the molecules, evaporated from a homemade Knudsen cell, on the crystal kept at room temperature. The sublimation temperatures were $770 \mathrm{~K}$ and $670 \mathrm{~K}$ for PdPc and
PtPc, respectively. A multilayer phase, which does not exhibit a LEED pattern, is prepared by sublimating PtPc more than ten times longer than the sublimation time necessary to obtain the ordered monolayer phase. The pressure in the preparation chamber did not exceed $1 \times 10^{-8}$ mbar during sublimation.

After the layer was prepared, its long-range order was checked by LEED. Vibrational features were recorded in HREELS with a primary electron beam energy of $6.3 \mathrm{eV}$ in both specular and off-specular geometries. The incoming beam is directed to the sample with an angle of $45^{\circ}$ with respect to surface normal. In the specular (off-specular) geometry the electrons are detected at an angle of $45^{\circ}\left(60^{\circ}\right)$ with respect to the surface normal. The energy resolution, estimated from the full width at half maximum (FWHM) of the elastic peak, is in the range of $16-22 \mathrm{~cm}^{-1}(2-2.7 \mathrm{meV})$. In order to interpret the complex vibrational spectra, we performed theoretical calculations of isolated PtPc and PdPc molecules using GAUSSIAN [26]. The DFT calculations of their electronic structure and of their vibrational eigenfrequencies were carried out using the LanL2DZ basis set and the B3LYP functional. The calculated vibration energies were compressed by a factor of 0.9456 [27,28].

\section{PLATINUM-PHTHALOCYANINE ON Ag(111)}

\section{A. Long-range order}

PtPc molecules form ordered two-dimensional structures on the $\operatorname{Ag}(111)$ surface. Like for most of the MPc molecules, two diffuse rings are detected at room temperature in LEED at low coverage after annealing a thick layer up to $730 \mathrm{~K}$ (see Ref. [29]). This two-dimensional disordered phase has already been observed in the case of, for example, CuPc on $\mathrm{Ag}(111)$ and $\mathrm{Au}(111)[24,30,31]$. Stadler et al. interpreted the presence of this two-dimensional gas phase at low coverage as the result of an intermolecular repulsion [32]. As the density of the PtPc molecules on the surface increases, diffraction spots are detected in LEED at room temperature (ordered phase). According to high-resolution structural measurements for $\mathrm{CuPc}$, the unit cell formed by the $\mathrm{CuPc}$ molecules decreases continuously in size as the coverage is increased. Our LEED measurements indicate a similar behavior for $\mathrm{PtPc}$ on $\mathrm{Ag}(111)$.

\section{B. Vibrational properties of the multilayer and mode assignment}

PtPc has $3 N-6=165$ distinct vibrational modes ( $N=57$ is the number of atoms in the molecule). Its point group is $D_{4 h}$ and its vibrational representation is $\Gamma=14 A_{1 \mathrm{~g}}+13 A_{2 \mathrm{~g}}+14 B_{1 \mathrm{~g}}+14 B_{2 \mathrm{~g}}+26 E_{\mathrm{g}}+56 E_{\mathrm{u}}+$ $8 A_{2 \mathrm{u}}+6 A_{1 \mathrm{u}}+7 B_{1 \mathrm{u}}+7 B_{2 \mathrm{u}}$. Modes transforming according to the irreducible representations $A_{2 \mathrm{u}}$ and $E_{\mathrm{u}}$ are IR-active modes; i.e., the motion of the atoms produces a dynamic dipole moment $\vec{\mu}_{\mathrm{dyn}}$. Depending on the direction of $\vec{\mu}_{\mathrm{dyn}}$, the IR-active modes can be classified as in-plane modes, in which $\vec{\mu}_{\text {dyn }}$ lies in the molecular $x y$ plane $\left[E_{\mathrm{u}}(x, y)\right.$ modes], and as out-of-plane modes, in which $\vec{\mu}_{\mathrm{dyn}}$ is oriented perpendicular to the molecular plane along the $z$ direction $\left[A_{2 \mathrm{u}}(z)\right.$ modes]. The modes belonging to the $A_{1 \mathrm{~g}}, E_{\mathrm{g}}, B_{1 \mathrm{~g}}$, and $B_{2 \mathrm{~g}}$ irreducible representations are Raman (R) active modes; i.e., the atomic vibration induces a change of the molecular polarizability. 


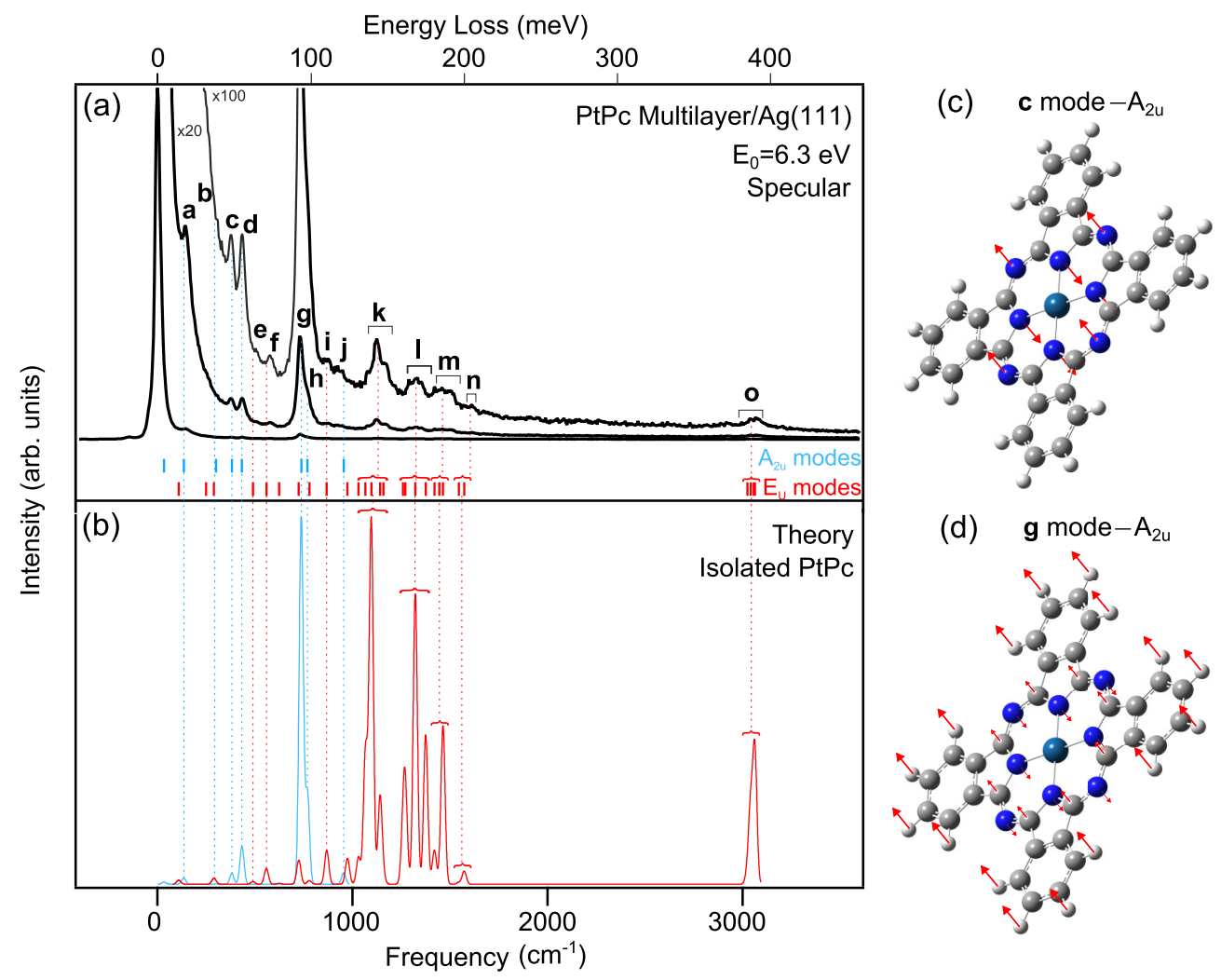

FIG. 1. (a) The experimental specular HREELS spectrum of the PtPc multilayer on $\mathrm{Ag}(111)$ and (b) the calculated spectrum of the isolated molecule are shown. The calculated vibrational frequencies of the out-of-plane $A_{2 \mathrm{u}}$ and in-plane $E_{\mathrm{u}}$ IR-active modes are displayed in blue and red, respectively. The atomic displacements of the $\mathbf{c}$ mode and $\mathbf{g}$ mode are shown in (c) and (d), respectively.

We refer the reader to Ref. [33] for an introduction to symmetry-based selection rules.

In this work we are interested in IR-active modes, because HREELS is sensitive to the dynamic dipole moment $\vec{\mu}_{\text {dyn }}$. According to the surface selection rule [25], modes for which $\vec{\mu}_{\text {dyn }}$ is oriented perpendicular to the surface are excited in the dipole scattering mechanism (specular geometry), whereas modes having a dynamic dipole oriented parallel to the surface are suppressed, because the $\vec{\mu}_{\text {dyn }}^{*}$ produced by the image charges in the substrate is antiparallel to $\vec{\mu}_{\text {dyn }}$, leading to a cancellation of the two. In the language of group theory, the surface selection rule states that only totally symmetric modes $\left(A_{1}, A^{\prime}\right.$, and $A$ representations) of the moleculesubstrate complex may be visible in HREELS. In contrast, all modes can be excited in the impact scattering regime (off-specular geometry). The appearance and/or disappearance of specific modes in the HREELS spectrum, depending on the measurement geometry, gives important information on the symmetry of the molecule-substrate complex. Therefore, an accurate assignment of the vibrational features is necessary.

The assignment is carried out by comparing the experimental peaks in the spectrum of a multilayer with calculated frequencies of the isolated PtPc molecule. In the multilayer, the effect of the substrate is reduced and thus the spectral properties are expected to be similar to the isolated molecule. Figure 1(a) shows the experimental spectrum of a PtPc multilayer on $\operatorname{Ag}(111)$, acquired in specular geometry, in comparison with the calculated spectrum of the isolated PtPc molecule [Fig. 1(b)]. The calculated spectrum contains a Gaussian broadening of $20 \mathrm{~cm}^{-1}$, similar to the experimentally observed one, and is composed of $A_{2 \mathrm{u}}$ (blue lines) and $E_{\mathrm{u}}$ (red lines) modes only, because these are the sole IRactive vibrations. We note that all experimental features can be identified in good agreement with theory. A simplified description of the modes is given in Table I (see Ref. [29] for details). The spectrum is dominated by the $A_{2 \mathrm{u}}$ modes, with dynamic dipole moments perpendicular to the molecular plane, such as the strongest vibrations a at $145 \mathrm{~cm}^{-1}$ (out-of-plane bending of the whole molecule except $\mathrm{Pt}$ ) and $\mathbf{g}$ at $730 \mathrm{~cm}^{-1}$ [out-of-plane bending of the central ring of alternating $\mathrm{C}$ and $\mathrm{N}$ atoms around the metal porphyrazin macrocycle together with the $\mathrm{H}$ atoms; Fig. 1(d)]. Compared to theory, the experimental $E_{\mathrm{u}}$ mode intensities appear reduced, suggesting a predominantly flat adsorption orientation of the molecules in the multilayer. However, the intensity ratio of the in-plane modes $\left(E_{\mathrm{u}}\right)$ and out-of-plane modes $\left(A_{2 \mathrm{u}}\right)$ is conserved in the off-specular spectrum (not shown). This can be caused by a residual contribution of the dipole scattering in the off-specular geometry due to, e.g., surface roughness.

\section{Vibrational properties of the ordered monolayer phase and molecular symmetry reduction}

The vibrational properties of the ordered monolayer phase are discussed in comparison with those of the multilayer and calculated frequencies. Experimental modes are assigned to 
TABLE I. List of the experimental vibrational modes present in the PtPc multilayer and ordered monolayer phase specular spectra on $\operatorname{Ag}(111)$ accompanied by their irreducible representation and description. The calculated frequencies are compressed by a factor of 0.9456 . The abbreviations are as follows: $\mathrm{OP}=$ out of plane, $\mathrm{IP}=$ in plane, bend = bending mode, deform $=$ deformation mode, sciss $=$ scissoring mode, stretch $=$ stretching mode, rock $=$ rocking mode. All values are given in $\mathrm{cm}^{-1}$.

\begin{tabular}{|c|c|c|c|c|c|c|}
\hline \multirow{3}{*}{$\begin{array}{l}\text { Modes } \\
\mathbf{a} \\
\text { R1 }\end{array}$} & \multirow{2}{*}{$\begin{array}{c}\text { Multilayer } \\
145\end{array}$} & \multirow{3}{*}{$\begin{array}{c}\text { Ordered monolayer } \\
145 \\
255\end{array}$} & \multirow{2}{*}{$\begin{array}{l}\text { DFT } \\
136\end{array}$} & \multirow{2}{*}{$\begin{array}{c}\text { Irreducible representation } \\
A_{2 \mathrm{u}}\end{array}$} & \multicolumn{2}{|c|}{ Description } \\
\hline & & & & & OP bend: & Molecule \\
\hline & & & & & & \\
\hline b & 310 & & 301 & $A_{2 \mathrm{u}}$ & OP bend: & Molecule \\
\hline c & 380 & 350 & 382 & $A_{2 \mathrm{u}}$ & OP bend: & $\mathrm{N}$ atoms \\
\hline d & 430 & 430 & 434 & $A_{2 \mathrm{u}}$ & OP bend: & $\mathrm{N}+\mathrm{C}$ atoms \\
\hline $\mathbf{e}$ & 505 & & 490 & $E_{\mathrm{u}}$ & IP sciss: & $\mathrm{N}+\mathrm{C}$ atoms \\
\hline $\mathbf{R} 2$ & & 565 & & & & \\
\hline $\mathbf{f}$ & 575 & & 574 & $E_{\mathrm{u}}$ & IP deform: & Molecule \\
\hline $\mathbf{R 3}$ & & 655 & & & & \\
\hline $\mathbf{g}$ & 730 & 715 & 738 & $A_{2 \mathrm{u}}$ & OP bend: & Main ring \\
\hline h & 765 & 765 & 770 & $A_{2 \mathrm{u}}$ & OP bend: & $\mathrm{N}$ atoms + Phenyl groups \\
\hline i & 880 & & 869 & $E_{\mathrm{u}}$ & IP deform: & Molecule \\
\hline $\mathbf{j}$ & 925 & & 956 & $A_{2 \mathrm{u}}$ & OP bend: & Phenyl groups \\
\hline k & $1075-1200$ & & $1031-1159$ & $E_{\mathrm{u}}$ & IP deform/stretch: & Molecule \\
\hline F1 & & $1075-1200$ & & & & \\
\hline 1 & $1285-1375$ & & $1260-1323$ & $E_{\mathrm{u}}$ & IP rock/stretch: & $\mathrm{N}$ atoms + Phenyl groups \\
\hline F2 & & $1285-1375$ & & & & \\
\hline $\mathbf{m}$ & $1410-1530$ & & $1376-1465$ & $E_{\mathrm{u}}$ & IP stretch: & Main ring \\
\hline F3 & & $1410-1530$ & & & & \\
\hline $\mathbf{n}$ & $1585-1640$ & & $1546-1574$ & $E_{\mathrm{u}}$ & IP stretch: & Phenyl groups \\
\hline $\mathbf{o}$ & $3010-3030$ & & $3025-3064$ & $E_{\mathrm{u}}$ & IP stretch: & $\mathrm{H}$ atoms \\
\hline
\end{tabular}

calculated ones within $50 \mathrm{~cm}^{-1}$ of their centroids, a range that corresponds to the typical experimental width of isolated peaks (see semitransparent blue boxes in Fig. 2 and Fig. 4). Figure 2(a) shows specular HREELS spectra of the ordered monolayer phase and the multilayer. In the ordered monolayer, most of the $E_{\mathrm{u}}$ in-plane modes are absent, especially between 1000 and $1600 \mathrm{~cm}^{-1}$. But in this range three asymmetric peaks $(\mathbf{F 1}, \mathbf{F 2}$, and $\mathbf{F 3})$ are detected. Their origin will be discussed later. The absence of the in-plane vibrations indicates that the surface selection rule strongly attenuates the in-plane $E_{\mathrm{u}}$ modes in comparison to the out-of-plane $A_{2 \mathrm{u}}$ modes. We conclude that the molecules in the monolayer are oriented strictly parallel to the Ag surface. Note that the $A_{2 u}$ peaks $\mathbf{b}$ and $\mathbf{j}$, of very weak intensity in the multilayer (Fig. 1), are not detectable in the ordered monolayer phase [Fig. 2(a)] in agreement with results on other phthalocyanines [34-36].

\section{Out-of-plane $A_{2 \mathrm{u}}$ modes}

Like in the multilayer, all of the out-of-plane $A_{2 \mathrm{u}}$ modes are detected in the spectrum of the ordered monolayer phase (except $\mathbf{b}$ and $\mathbf{j}$ ). However, in the latter the $\mathbf{c}$ and $\mathbf{g}$ modes are shifted to lower wave numbers. The shift of the $\mathbf{c}$ mode $\left(\simeq 30 \mathrm{~cm}^{-1}\right)$ is larger than that of the $\mathrm{g}$ mode $\left(\simeq 15 \mathrm{~cm}^{-1}\right)$. The $\mathbf{c}$ mode is associated with the out-of-plane bending of the aza bridge $\mathrm{N}$ atoms against the pyrrole $\mathrm{N}$ atoms [Fig. 1(c)], while in the $\mathbf{g}$ mode all $\mathrm{N}$ atoms move in phase against the $\mathrm{C}$ atoms in the porphyrazin macrocycle and the $\mathrm{H}$ atoms in the phenyl groups [Fig. 1(d)]. We assign the sizable shifts of these two modes to the molecule-substrate interaction. In contrast to FePc and $\mathrm{CoPc}$ on $\mathrm{Ag}(111)$ [37,38], for which the central metal forms a covalent bond with the substrate, the molecule-substrate interaction in PtPc takes place through the porphyrazin macrocycle, because neither the $\mathbf{c}$ nor the $\mathbf{g}$ modes, which are the ones that are most strongly influenced by the substrate, involve the central metal atom, unlike the a mode for example, which involves the metal atom but does not show an appreciable shift. This observation is in agreement with the general trend of a reduced metal participation in the moleculesubstrate bond as the number of $d$-electrons increases [4]. The observation that the shift of $\mathbf{c}$ is larger than that of $\mathbf{g}$, in conjunction with the fact that the $\mathbf{c}$ mode displacement is due to the $\mathrm{N}$ atoms only while in the $\mathbf{g}$ mode the displacements of $\mathrm{N}$ atoms are relatively small, suggests that most of the interaction between the molecule and the Ag substrate occurs via the $\mathrm{N}$ atoms.

\section{Raman modes}

Another noteworthy difference between the vibrational properties of the ordered monolayer phase and the multilayer is the appearance of new modes labeled $\mathbf{R 1}, \mathbf{R 2}$, and $\mathbf{R 3}$ in Fig. 2(a). Employing a range of $50 \mathrm{~cm}^{-1}$ around their centroids, the $\mathbf{R} 1$ peak at $255 \mathrm{~cm}^{-1}$ can be interpreted either as a $E_{\mathrm{u}}, A_{1 \mathrm{~g}}$ or as a $E_{\mathrm{g}}$ mode, while the $\mathbf{R} 2$ peak at $565 \mathrm{~cm}^{-1}$ may be attributed to either a $E_{\mathrm{u}}$ or a $A_{1 \mathrm{~g}}$ mode. Finally, the $\mathbf{R} 3$ peak at $655 \mathrm{~cm}^{-1}$ can either be ascribed to a $A_{1 \mathrm{~g}}$ or a $B_{2 \mathrm{~g}}$ mode. The possible assignments are summarized in Table III. Ruling out the $E_{\mathrm{u}}$ modes, because as in-plane IR modes they should be screened by the metal surface, only modes having an $A_{1 \mathrm{~g}}, E_{\mathrm{g}}$, or $B_{2 \mathrm{~g}}$ irreducible representation in the $D_{4 h}$ point group remain as possible assignments. However, they are R-active modes and as such not expected to contribute to the spectrum of an isolated 


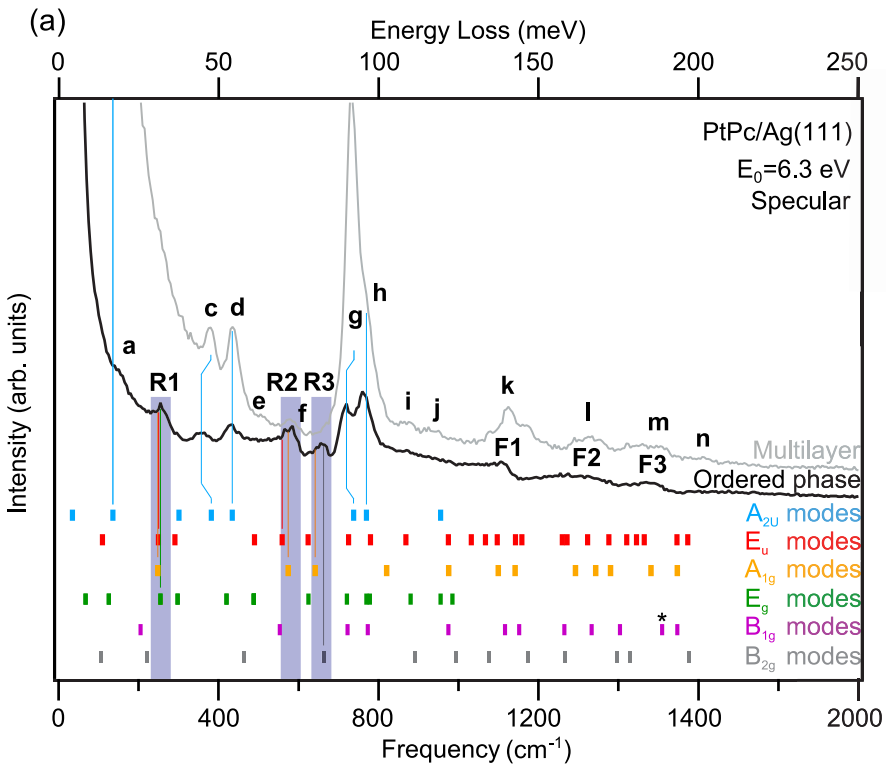

(b) $\quad$ LUMO $-\mathrm{E}_{\mathrm{g}} \rightarrow \mathrm{B}_{1}+\mathrm{B}_{2}$

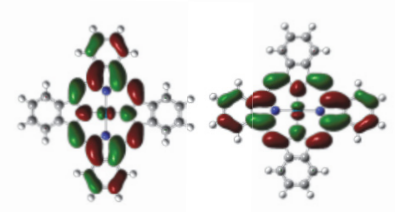

(c) F3 mode $-B_{1 g} \rightarrow A_{1}\left(^{*}\right)$

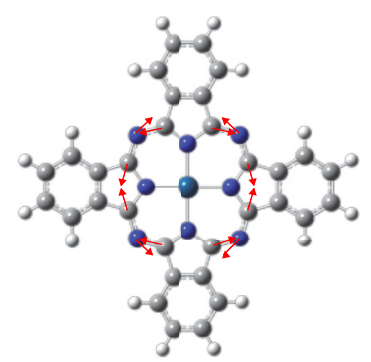

FIG. 2. (a) The comparison of the specular HREELS spectra of the PtPc ordered monolayer phase and multilayer on Ag(111). The simulated vibrational modes of the isolated molecule are indicated as thick colored vertical bars. The energy ranges used for assignment around the centroids of $\mathbf{R} 1, \mathbf{R 2}$, and $\mathbf{R 3}$ are depicted as semitransparent blue boxes, and possible assignments are indicated by thin vertical bars. Note that the vibrational properties of the ordered monolayer phase do not change with the coverage. (b) The calculated charge density of the two degenerate LUMOs of isolated PtPc. (c) Sketch of the Raman mode belonging to the $B_{1 \mathrm{~g}}$ irreducible representation at $1509 \mathrm{~cm}^{-1}[\mathrm{marked}$ with * in panel (a)] that is used to fit the Fano peak $\mathbf{F 3}$ at approximately $1500 \mathrm{~cm}^{-1}$ (see text).

molecule. But they can be activated if the molecular symmetry is reduced. Thus, a symmetry reduction of the molecule in contact with the surface must be considered.

When a PtPc molecule adsorbs with its plane parallel to the surface, its molecular symmetry is reduced from the original $D_{4 h}$ point group. Specifically, at least the in-surface-plane $(x, y)$ mirror symmetry is broken. A PtPc molecule lacking its $(x, y)$-plane symmetry belongs to the $C_{4 v}$ point group. However, further symmetry reductions are possible, e.g., to the $C_{2 v}$ point group, if the fourfold symmetry of the molecule is broken. With the help of so-called correlation tables it is possible to deduce how the modes (irreducible representations) of the isolated molecule $\left(D_{4 h}\right)$ relate to the ones of the molecule-substrate system of reduced symmetry $\left(C_{4 v}\right.$ or lower) [25]. An adaptation of the correlation tables of the $D_{4 h}$ point group from Ref. [25], considering the observed $\mathbf{R}$ modes (and the $\mathbf{F}$ modes, introduced in the next section), is given in Table II.

In principle, there is a chance that the observation of the $\mathrm{R}$ modes allows us to deduce the symmetry of the molecule-substrate complex. For example, a $B_{2 \mathrm{~g}}$ mode would become activated for HREELS dipole scattering only if the $D_{4 h}$ symmetry was reduced to $C_{2 v}\left(\sigma_{d}\right), C_{s}\left(\sigma_{d}\right)$, or $C_{2}$ upon adsorption (Table II). Hence, we must look for a single reduced point group in which the vibrational modes of the free molecule which we assign to $\mathbf{R} \mathbf{1}, \mathbf{R 2}$, and $\mathbf{R 3}$ each map onto a

TABLE II. Correlation table of the $D_{4 h}$ point group limited to the possible modes assignment of the $\mathbf{R}$ and $\mathbf{F}$ modes [25,33,39]. The notations $x, y$, and $z$ denote linear functions which transform according to the given irreducible representations. The other irreducible representations are not IR active.

\begin{tabular}{lcccccc}
\hline \hline$D_{4 h}$ & $C_{4 v}$ & $C_{2 v}\left(\sigma_{v}\right)$ & $C_{s}\left(\sigma_{v}=m_{x}\right)$ & $C_{s}\left(\sigma_{v}=m_{y}\right)$ & $C_{4}$ & $C_{2}$ \\
\hline$A_{1 \mathrm{~g}}$ & $A_{1}(z)$ & $A_{1}(z)$ & $A^{\prime}(z)$ & $A^{\prime}(z)$ & $A(z)$ & $B(z)$ \\
$B_{1 \mathrm{~g}}$ & $B_{1}$ & $A_{1}(z)$ & $A^{\prime}(z)$ & $A^{\prime}(z)$ & $B(z)$ & $A^{\prime \prime}(y)$ \\
$B_{2 \mathrm{~g}}$ & $B_{2}$ & $A_{2}$ & $A^{\prime \prime}(x)$ & $A^{\prime}(x)+A^{\prime \prime}(y)$ & $E(x+i y)+E(x-i y)$ & $B(x)+B(y)$ \\
$E_{\mathrm{g}}$ & $E(x, y)$ & $B_{1}(x)+B_{2}(y)$ & $A^{\prime \prime}(x)+A^{\prime}(y)$ & $A^{\prime}(x)+A^{\prime \prime}(y)$ & $E(x+i y)+E(x-i y)$ & $B(x)+B(y)$ \\
$E_{\mathrm{u}}(x, y)$ & $E(x, y)$ & $B_{1}(x)+B_{2}(y)$ & $A^{\prime \prime}(x)+A^{\prime}(y)$ & $C_{s}\left(\sigma_{d}=m_{d^{\prime}}\right)$ & \\
\hline$D_{4 h}$ & $C_{2 v}\left(\sigma_{d}\right)$ & $C_{s}\left(\sigma_{d}=m_{d}\right)$ & $A^{\prime}(z)$ & \\
\hline$A_{1 \mathrm{~g}}$ & $A_{1}(z)$ & $A^{\prime}(z)$ & $A^{\prime \prime}(x+y)$ & \\
$B_{1 \mathrm{~g}}$ & $\left.A_{2}+y\right)$ & $A^{\prime}(z)$ & \\
$B_{2 \mathrm{~g}}$ & $A_{1}(z)$ & $A^{\prime \prime}(x-y)$ & $A^{\prime}(z)$ & \\
$E_{\mathrm{g}}$ & $B_{1}(x+y)+B_{2}(x-y)$ & $A^{\prime}(x+y)+A^{\prime \prime}(x-y)$ & $A^{\prime \prime}(x+y)+A^{\prime}(x-y)$ & \\
$E_{\mathrm{u}}(x, y)$ & $B_{1}(x+y)+B_{2}(x-y)$ & $A^{\prime}(x+y)+A^{\prime \prime}(x-y)$ & $A^{\prime \prime}(x+y)+A^{\prime}(x-y)$ & \\
\hline \hline
\end{tabular}


TABLE III. Possible assignments of the $\mathbf{R}$ and $\mathbf{F}$ modes observed in the ordered monolayer phase of PtPc on $\operatorname{Ag}(111)$. The $\mathbf{F}$ mode assignments are based on the fit results presented in the Supplemental Material [29].

\begin{tabular}{lc}
\hline \hline Mode & Symmetry \\
\hline $\mathbf{R} 1$ & $A_{1 \mathrm{~g}} / E_{\mathrm{u}}(x, y) / E_{\mathrm{g}}$ \\
$\mathbf{R 2}$ & $A_{1 \mathrm{~g}} / E_{\mathrm{u}}(x, y)$ \\
$\mathbf{R 3}$ & $A_{1 \mathrm{~g}} / B_{2 \mathrm{~g}}$ \\
$\mathbf{F 1}$ & $A_{1 \mathrm{~g}} / E_{\mathrm{u}}(x, y) / B_{1 \mathrm{~g}}$ \\
$\mathbf{F 3}$ & $B_{1 \mathrm{~g}}$ \\
\hline \hline
\end{tabular}

totally symmetric representation ( $A_{1}, A^{\prime}$, or $A$; see Ref. [39]). However, since it is a possibility that $\mathbf{R 1}, \mathbf{R 2}$, and $\mathbf{R 3}$ could all originate from $A_{1 \mathrm{~g}}$ modes (see Table III), all three would in this case be visible in dipole scattering HREELS irrespective of the symmetry of the molecule-substrate complex $\left(C_{4 v}, C_{2 v}\right.$, $C_{4}, C_{s}$, or $C_{2}$ ). Hence, it is not possible to conclude on the basis of R1, R2, and R3 alone whether PtPc preserves its fourfold symmetry upon adsorption $\left(C_{4 v}\right)$ or lowers its symmetry $\left(C_{2 v}\right.$, $C_{4}, C_{s}$, or $C_{2}$ ).

\section{Asymmetric peaks}

In order to see whether the symmetry of the PtPc molecule on the Ag surface can be identified, we now turn to the analysis of the $\mathbf{F}$ modes. Between 1000 and $1600 \mathrm{~cm}^{-1}$, where the $E_{\mathrm{u}}$ modes are excited in the multilayer spectrum [Fig. 1(a)], three asymmetric features, labeled F1, F2, and F3 in Fig. 2(a), are visible in the spectrum of the ordered monolayer. Asymmetric line shapes of Fano type are well known for molecular and atomic adsorbates on metallic surfaces in the presence of an IDCT [34,35,40-45]. Since we are going to employ both IDCT selection rules and line shape analysis in our argument, we briefly sketch out their theoretical basis.

A prerequisite for the occurrence of IDCT is an electronvibron coupling that breaks the Born-Oppenheimer approximation according to which electronic degrees of freedom are decoupled from vibrational degrees of freedom, obeying their own separate electronic Schrödinger equation. While in the vibrational Schrödinger equation the energy eigenvalues of the electronic Schrödinger equation always serve as potential energy terms for the nuclear degrees of freedom, in the BornOppenheimer approximation the dependence of the electronic Schrödinger equation on nuclear coordinates is usually taken as being merely parametric.

In contrast, the explanation of IDCT requires the presence of a term in the Hamiltonian of the electron system that makes its dependence on vibrational coordinates explicit. In linear Taylor expansion, this electron-vibron coupling term has the form

$$
E\left(\left\{Q_{\alpha 0}+\Delta Q_{\alpha}\right\}\right)=E_{0}+\sum_{\alpha}\left(\frac{\partial E\left(\left\{Q_{\alpha}\right\}\right)}{\partial Q_{\alpha}}\right)_{0} \Delta Q_{\alpha}+\cdots
$$

Here $E_{0}=E\left(\left\{Q_{\alpha 0}\right\}\right)$. In the following we set $Q_{\alpha 0}=0$ and thus $\Delta Q_{\alpha}=Q_{\alpha}$. Moreover, we focus on a single normal vibrational mode $Q_{\alpha}$. Then the relevant coupling term in the
Hamiltonian becomes

$$
\hat{H}_{\alpha}^{\mathrm{el}-\mathrm{vib}}=\sqrt{\frac{\hbar}{2 \omega_{\alpha}}} \frac{\partial E\left(\left\{Q_{\alpha}\right\}\right)}{\partial Q_{\alpha}}\left(b_{\alpha}+b_{\alpha}^{\dagger}\right),
$$

where we have inserted the second quantized expression for the vibrational mode $Q_{\alpha}$ [39], and where $b_{\alpha}\left(b_{\alpha}^{\dagger}\right)$ is the annihilation (creation) operator of a vibrational quantum of mode $\alpha$.

To see whether the coupling term in Eq. (2) is allowed by symmetry we calculate its matrix element and analyze under which conditions it may be nonvanishing [39]. In calculating the matrix element we use wave functions $\Psi=|\psi\rangle\left|n_{\alpha}\right\rangle$ that consist of an electronic part $|\psi\rangle$ and a vibrational part $\left|n_{\alpha}\right\rangle$, where $n_{\alpha}$ designates the occupancy of vibrational mode $Q_{\alpha}$. The matrix element then reads

$$
M_{\alpha}=\left|\left\langle\Psi_{\mathrm{f}}\left|\hat{H}_{\alpha}^{\mathrm{el}-\mathrm{vib}}\right| \Psi_{\mathrm{i}}\right\rangle\right|^{2},
$$

where the indices denote initial and final states. Inserting the wave functions in $M_{\alpha}$ one finds

$$
M_{\alpha} \propto \sqrt{\frac{\hbar}{2 \omega_{\alpha}}} s_{\alpha}\left|\left\langle\psi_{\mathrm{f}}\left|\frac{\partial E\left(\left\{Q_{\alpha}\right\}\right)}{\partial Q_{\alpha}}\right| \psi_{\mathrm{i}}\right\rangle\right|^{2}
$$

for processes involving a single vibrational quantum, where $s_{\alpha}=n_{\alpha}\left(s_{\alpha}=n_{\alpha}+1\right)$ for the absorption (emission) of a vibrational quantum. Only if the right-hand side of Eq. (4) transforms totally symmetric under all symmetry operations of the system's Hamiltonian can $M_{\alpha}$ have a nonvanishing value.

Let us denote the totally symmetric irreducible representation of the point group $\mathrm{G}$ of the Hamiltonian as $\Gamma_{\mathrm{ts}}$ (depending on the particular $\mathrm{G}$ under investigation, this may be $A_{1}, A_{1 \mathrm{~g}}$, $A_{\mathrm{g}}, A_{1}^{\prime}, A^{\prime}$, or $A$; see Ref. [39]). Clearly, the energy $E$ in Eq. (4) has to have the full symmetry of the Hamiltonian, i.e., must transform according to $\Gamma_{\text {ts. }}$. The vibrational mode $Q_{\alpha}$, being an eigenstate of the vibrational Hamiltonian, transforms as the irreducible representation $\Gamma_{\alpha}$ of $\mathrm{G}$. The same is thus true of the partial derivative $\partial E\left(\left\{Q_{\alpha}\right\}\right) / \partial Q_{\alpha}$. As eigenstates of the electronic Hamiltonian the wave functions $\psi_{\mathrm{i}}$ and $\psi_{\mathrm{f}}$ transform according to $\Gamma_{\mathrm{i}}$ and $\Gamma_{\mathrm{f}}$, respectively, both of which are irreducible representations of $\mathrm{G}$, too. Hence, the matrix element $M_{\alpha}$ transforms according to the direct product representation $\Gamma_{M_{\alpha}}=\Gamma_{\mathrm{f}} \otimes \Gamma_{\alpha} \otimes \Gamma_{\mathrm{i}}$. As a rule, $\Gamma_{M_{\alpha}}$ is reducible, and only if the reduction of $\Gamma_{M_{\alpha}}$ contains $\Gamma_{\text {ts }}$ can $M_{\alpha}$ be nonzero. The condition for $M_{\alpha} \neq 0$ thus is

$$
\Gamma_{\mathrm{f}} \otimes \Gamma_{\alpha} \otimes \Gamma_{\mathrm{i}} \supseteq \Gamma_{\text {ts }} .
$$

According to a well-known theorem of group theory this can only happen if the reduction of the direct product of any pair of irreducible representations on the left of Eq. (5) contains the third [46]. For instance,

$$
\Gamma_{\mathrm{f}} \otimes \Gamma_{\mathrm{i}} \supseteq \Gamma_{\alpha}
$$

must hold. At this stage we deploy additional information regarding the vibrational mode $Q_{\alpha}$. Since we excite this mode via dipole scattering in electron energy loss spectroscopy, the surface selection rule applies. Thus $\Gamma_{\alpha}=\Gamma_{\mathrm{ts}}$, i.e., the vibration must be totally symmetric. As an immediate consequence, $\Gamma_{\mathrm{f}} \supseteq \Gamma_{\mathrm{i}}$ follows from Eq. (6) because of the above mentioned theorem, which means $\Gamma_{\mathrm{f}}=\Gamma_{\mathrm{i}}$, since $\Gamma_{\mathrm{f}}$ and $\Gamma_{\mathrm{i}}$ are irreducible. In other words, initial and final states $\psi_{i}$ and $\psi_{f}$ of the electron involved in the coupling may transform according to 
an arbitrary irreducible representation, provided they are the same. Obviously, this condition is trivially fulfilled if $\psi_{\mathrm{i}}=\psi_{\mathrm{f}}$. While in an electron-vibron scattering process, occurring with an electron of the material, $\psi_{\mathrm{i}}$ and $\psi_{\mathrm{f}}$ cannot be equal, since this would contradict the conservation of energy $E_{\mathrm{i}}=E_{\mathrm{f}}+\omega_{\alpha}$ for vibron emission or $E_{\mathrm{f}}=E_{\mathrm{i}}+\omega_{\alpha}$ for vibron absorption (note that we set $\hbar=1$ from now on), in the present case, where the energy for the excitation of the vibron is provided completely by the probing electron, $\psi_{\mathrm{i}}=\psi_{\mathrm{f}}$ is in fact necessarily fulfilled for reasons of energy conservation (disregarding an accidental degeneracy between electronic states $\psi_{\mathrm{i}}$ and $\psi_{\mathrm{f}}$ ). We can thus conclude that for totally symmetric vibrations taking part in IDCT the matrix element $M_{\alpha}$ in Eq. (4) is symmetry-allowed for electronic levels that transform according to an arbitrary irreducible representation.

In addition to the participation of a totally symmetric vibrational mode, a further prerequisite of IDCT is the partial filling of the orbital with energy $E_{\mathrm{i}}$ that couples to the molecular vibration in question. Then, as the vibration is excited, the associated distortion of the molecule along the vibrational coordinate modulates the orbital energy periodically, leading to the excitations of electron hole pairs and oscillatory charge flow between the molecule and the substrate that is effectively pumped by the vibration.

We now turn to the line shape of modes taking part in interfacial dynamical charge transfer. The line shape of the vibration as measured in electron energy loss spectroscopy follows from the loss function $-\operatorname{Im} \epsilon^{-1}$, where $\epsilon$ is the dielectric response function. Since $\epsilon$ is given by $\epsilon(\omega)=$ $\epsilon_{\infty}+4 \pi \chi(\omega)\left(\epsilon_{\infty}\right.$ is the background dielectric constant), one must calculate the generalized susceptibility $\chi(\omega)=\delta \hat{n} / E_{\text {ext }}$ that determines the dynamic charge transfer response $\delta \hat{n}$ to the electric field $E_{\text {ext }}$ of the dipole-scattered probing electron. Based on the linear expansion in Eq. (1), $\chi(\omega)$ is given by [42]

$$
\chi(\omega)=\frac{\rho\left(E_{\mathrm{F}}\right)(e d)^{2} N \omega_{\mathrm{CT}}^{2}}{\omega_{\mathrm{CT}}^{2}\left(1-\lambda_{\alpha} D_{\alpha}\right)-\omega^{2}-i \omega \gamma_{\mathrm{CT}}} .
$$

$e$ is the elementary charge, $\omega_{\mathrm{CT}}$ is the eigenfrequency of the charge transfer oscillation (equivalent to the excitation of electron hole pairs by the modulation of the orbital energy $\left.E_{i}\right), \gamma_{\mathrm{CT}}$ its damping, $d$ the length scale of the dynamical dipole, $N$ the dipole density, $\lambda_{\alpha}=2 g_{\alpha}^{2} \rho\left(E_{\mathrm{F}}\right) / \omega_{\alpha}$ [where $g_{\alpha} \equiv \frac{1}{\sqrt{2}} \frac{\partial E_{i}}{\partial Q_{\alpha}}$ and $\rho\left(E_{\mathrm{F}}\right)$ is the density of states at the Fermi level] the dimensionless electron-vibron coupling parameter, $D_{\alpha}=\omega_{\alpha}^{2} /\left(\omega_{\alpha}^{2}-\omega^{2}-i \omega \gamma_{\alpha}\right)$, and $\gamma_{\alpha}$ and $\omega_{\alpha}$ are the damping and the eigenfrequency of the vibrational mode in the absence of coupling to electron-hole pairs, respectively. With Eq. (7), one can calculate the line shapes of vibrations that are subject to IDCT. This line shape turns out to be of Fano type [42].

We now show that the approach of Ref. [42] that leads to Eq. (7) is equivalent to the one adopted by Langreth [47]. To this end we analyze the vibrational line shape from a slightly different but fully equivalent point of view, focusing on the electronic dynamical dipole moment $\mu_{\alpha}^{\mathrm{el}}=\partial P / \partial Q_{\alpha}$ of vibration $Q_{\alpha}$. Specifically, we calculate the line shape of the vibration from the response of the polarization $P=N e d \delta \hat{n}$ [42] to the modulation of the electronic level energy $E_{i}$. This can be derived directly from Eq. (7), yielding

$$
\frac{\partial P}{\partial E_{i}}=-\frac{\omega_{\alpha}\left(\mu_{\alpha}^{\mathrm{el}}\right)^{2}}{\omega_{\alpha}^{2}\left(1-\lambda_{\alpha} D_{\mathrm{CT}}\right)-\omega^{2}-i \omega \gamma_{\alpha}},
$$

where $\mu_{\alpha}$ is the dynamical dipole moment of the vibration $Q_{\alpha}$ with frequency $\omega_{\alpha}$ and damping $\gamma_{\alpha}$ and $D_{\mathrm{CT}}=\omega_{\mathrm{CT}}^{2} /\left(\omega_{\mathrm{CT}}^{2}-\right.$ $\left.\omega^{2}-i \omega \gamma_{\mathrm{CT}}\right)$. In the limit $\omega \ll \omega_{\mathrm{CT}}$ and under the assumption that the electron-hole pair continuum that damps the vibration can be modeled by a single damped harmonic oscillator, whose frequency $\omega_{\mathrm{CT}}$ and damping $\gamma_{\mathrm{CT}}$ are related by $\omega_{\mathrm{CT}}=\gamma_{\mathrm{CT}} / 2$ (aperiodic limit), this becomes

$$
\frac{\partial P}{\partial E_{i}}=-\frac{\omega_{\alpha}\left(\mu_{\alpha}^{\mathrm{el}}\right)^{2}}{\bar{\omega}_{\alpha}^{2}-\omega^{2}-i \omega \bar{\gamma}_{\alpha}}
$$

with $\bar{\omega}_{\alpha}^{2}=\omega_{\alpha}^{2}\left(1-\lambda_{\alpha}\right)$ and $\bar{\gamma}_{\alpha}=\gamma_{\alpha}\left(1+\beta_{\alpha}\right)$. $\lambda_{\alpha}$ is the dimensionless electron-vibron coupling parameter introduced above and $\beta_{\alpha}=8 g_{\alpha}^{2} \omega_{\alpha} \rho\left(E_{\mathrm{F}}\right) / \gamma_{\alpha} \gamma_{\mathrm{CT}}$. Finally, if the position of the electronic level $E_{i}$ is approximately $\gamma_{\mathrm{CT}} / 2$ above the Fermi level $E_{\mathrm{F}}\left(\gamma_{\mathrm{CT}} \sim\left[\pi \rho\left(E_{\mathrm{F}}\right)\right]^{-1}\right)[47], \beta_{\alpha}$ becomes

$$
\beta_{\alpha}=\frac{8 \pi g_{\alpha}^{2} \omega_{\alpha} \rho\left(E_{\mathrm{F}}\right)^{2}}{\gamma_{\alpha}}=\lambda_{\alpha} \frac{\omega_{\alpha}^{2}}{\gamma_{\alpha}} 4 \pi \rho\left(E_{\mathrm{F}}\right) .
$$

As expected, the coupling of the vibration $Q_{\alpha}$ to the electron-hole pair continuum leads to a renormalization of the vibrational frequency $\omega_{\alpha} \rightarrow \bar{\omega}_{\alpha}$ and an increase of its damping $\gamma_{\alpha} \rightarrow \bar{\gamma}_{\alpha}$. However, as Langreth has pointed out [47], this is not its only effect. The very same mechanism that leads to the renormalization of $\omega_{\alpha}$ and $\gamma_{\alpha}$ will also lead to a complex dynamical dipole moment $\mu_{\alpha}^{\mathrm{el}}$ in Eq. (9) that changes the line shape of the vibration from a symmetric Lorentzian to an asymmetric Fano line shape.

From the equation for $\mu_{\alpha}^{\mathrm{el}}$ (Ref. [42])

$$
\mu_{\alpha}^{\mathrm{el}}=N e d \frac{\partial \hat{n}}{\partial Q_{\alpha}}=-\frac{\sqrt{2} g_{\alpha} N e d \rho\left(E_{\mathrm{F}}\right) \omega_{\mathrm{CT}}^{2}}{\omega_{\mathrm{CT}}^{2}-\omega^{2}-i \omega \gamma_{\mathrm{CT}}}
$$

we obtain in the limit $\omega \ll \omega_{\mathrm{CT}}$ the expressions

$$
\operatorname{Re} \mu_{\alpha}^{\mathrm{el}}=-\sqrt{2} g_{\alpha} N e d \rho\left(E_{\mathrm{F}}\right)
$$

and

$$
\operatorname{Im} \mu_{\alpha}^{\mathrm{el}}=-\sqrt{2} g_{\alpha} N e d \rho\left(E_{\mathrm{F}}\right) \omega \gamma_{\mathrm{CT}} / \omega_{\mathrm{CT}}^{2}
$$

for the real and imaginary parts of the dynamical dipole moment. If the electron-hole pair continuum that damps the vibration is modeled by a single damped harmonic oscillator in the aperiodic limit, as in the derivation of Eq. (9), one finds

$$
\operatorname{Im} \mu_{\alpha}^{\mathrm{el}}=-4 \pi \sqrt{2} g_{\alpha} N e d \rho\left(E_{\mathrm{F}}\right)^{2} \omega,
$$

which with Eq. (12) yields

$$
\frac{\operatorname{Im} \mu_{\alpha}^{\mathrm{el}}}{\operatorname{Re} \mu_{\alpha}^{\mathrm{el}}}=4 \pi \rho\left(E_{\mathrm{F}}\right) \omega .
$$

Equations (12)-(15) are analogous to the corresponding ones derived by Langreth [47].

If, as in the derivation of Eq. (10), we again assume that the position of the electronic level $E_{i}$ is approximately $\gamma_{\mathrm{CT}} / 2$ above the Fermi level $E_{\mathrm{F}}\left(\gamma_{\mathrm{CT}} \sim\left[\pi \rho\left(E_{\mathrm{F}}\right)\right]^{-1}\right)$, a particularly intuitive argument for the emergence of the imaginary part 
of the dynamical dipole moment becomes apparent. From Eq. (15) we find

$$
\frac{\operatorname{Im} \mu_{\alpha}^{\mathrm{el}}}{\operatorname{Re} \mu_{\alpha}^{\mathrm{el}}}=\frac{4 \omega}{\gamma_{\mathrm{CT}}},
$$

which at $\omega=\omega_{\alpha}$ becomes

$$
\frac{\operatorname{Im} \mu_{\alpha}^{\mathrm{el}}}{\operatorname{Re} \mu_{\alpha}^{\mathrm{el}}}=2 \pi \frac{\tau_{\mathrm{CT}}}{T_{\alpha}} .
$$

If the lifetime $\tau_{\mathrm{CT}} \equiv 4 / \gamma_{\mathrm{CT}}$ of the electronic level and therefore also of the electron-hole pair excitations is much shorter than the period $T_{\alpha}$ of a vibrational oscillation (i.e., $\tau_{\mathrm{CT}} \ll T_{\alpha}$ ), the electrons can follow the vibration adiabatically. According to Eq. (17), in this limit $\operatorname{Im} \mu_{\alpha}^{\mathrm{el}}$ is indeed negligible and $\mu_{\alpha}^{\mathrm{el}}$ is in phase with the vibration $Q_{\alpha}$ and accordingly also with $\mu_{\alpha}^{\text {ion }}$, the intrinsic dynamical dipole moment resulting from the motion of the ion cores. If, on the other hand, $\tau_{\mathrm{CT}} \gtrsim T_{\alpha}$, i.e., the lifetime of electron-hole pair excitations involving $E_{i}$ is comparable to or larger as the vibration period, $\operatorname{Im} \mu_{\alpha}^{\mathrm{el}}$ becomes appreciable and $\mu_{\alpha}^{\mathrm{el}}$, which is driven by the vibration at $\omega_{\alpha}$, lags behind $\mu_{\alpha}^{\text {ion }}$ in phase. This is the source of the additional vibrational damping beyond $\gamma_{\alpha}$ in Eq. (9) and leads to the nontrivial Fano line shape. Note that it is the lifetime of the electronic level, rather than the resonance frequency $\omega_{\mathrm{CT}}$ of the charge transfer oscillation, that defines the relevant time scale in Eq. (17).

Equation (9) can be used to calculate the line shape as $L(\omega)=-\operatorname{Im}\left[\partial P(\omega) / \partial E_{i}\right]$. Instead of using only the electronic dynamical dipole $\mu_{\alpha}^{\mathrm{el}}$ arising from the modulation of the electronic level (giving rise in turn to electron hole pair excitations), we employ $\mu_{\alpha}=\mu_{\alpha}^{\text {ion }}+\mu_{\alpha}^{\text {el }}$; i.e., we add the intrinsic dynamical dipole moment of the vibration that arises from the motion of the ion cores and for which $\mu_{\alpha}^{\text {ion }}=\operatorname{Re} \mu_{\alpha}^{\text {ion }}$. Then $\mu_{\alpha}=\operatorname{Re} \mu_{\alpha}(1+i \omega \tau)$ with

$$
\tau=\frac{\tau_{\mathrm{CT}}}{1+\operatorname{Re} \mu_{\alpha}^{\text {ion }} / \operatorname{Re} \mu_{\alpha}^{\mathrm{el}}}
$$

and we find [47-49]

$$
L(\omega)=c \bar{\omega}_{\alpha} \omega \bar{\gamma}_{\alpha} \frac{\left[1-\left(\omega^{2}-\bar{\omega}_{\alpha}^{2}\right) \tau / \bar{\gamma}_{\alpha}\right]^{2}}{\left(\omega^{2}-\bar{\omega}_{\alpha}^{2}\right)^{2}+\omega^{2} \bar{\gamma}_{\alpha}^{2}}-c \bar{\omega}_{\alpha} \omega \frac{\tau^{2}}{\bar{\gamma}_{\alpha}},
$$

where $c=\left(\operatorname{Re} \mu_{\alpha}\right)^{2} / \sqrt{1-\lambda_{\alpha}}$ is a constant. The small and linearly varying term in Eq. (19) is usually ignored, as it is canceled by an equal term of opposite sign that arises from the electronic polarizability [47].

We have used the first term in Eq. (19) to fit the $\mathbf{F}$ modes observed in our data. In the process we have treated $\tau$ and $\bar{\gamma}_{\alpha}$ as fit parameters, while DFT-calculated vibrational frequencies were used as input parameters for $\bar{\omega}_{\alpha}$. Note that it would be more correct to use the calculated frequencies as input for the unrenormalized $\omega_{\alpha}$. However, since typically $\lambda_{\alpha} \simeq 0.1$ (Ref. [42]), the renormalization is approximately 5\% and thus the incurred error is small. In some cases we have found negative $\tau$ as the result of the fitting. According to Eq. (18) the sign of $\tau$ is determined by the relative phases of $\mu_{\alpha}^{\text {ion }}, \operatorname{Re} \mu_{\alpha}^{\mathrm{el}}$, and $\operatorname{Im} \mu_{\alpha}^{\mathrm{el}}$ as rotating vectors in a polar diagram.

We now turn to our data in Fig. 3. In order to identify which vibrational modes are involved in the IDCT that produces F1, F2, and F3, Eq. (19) is used to fit these features, using values (a)

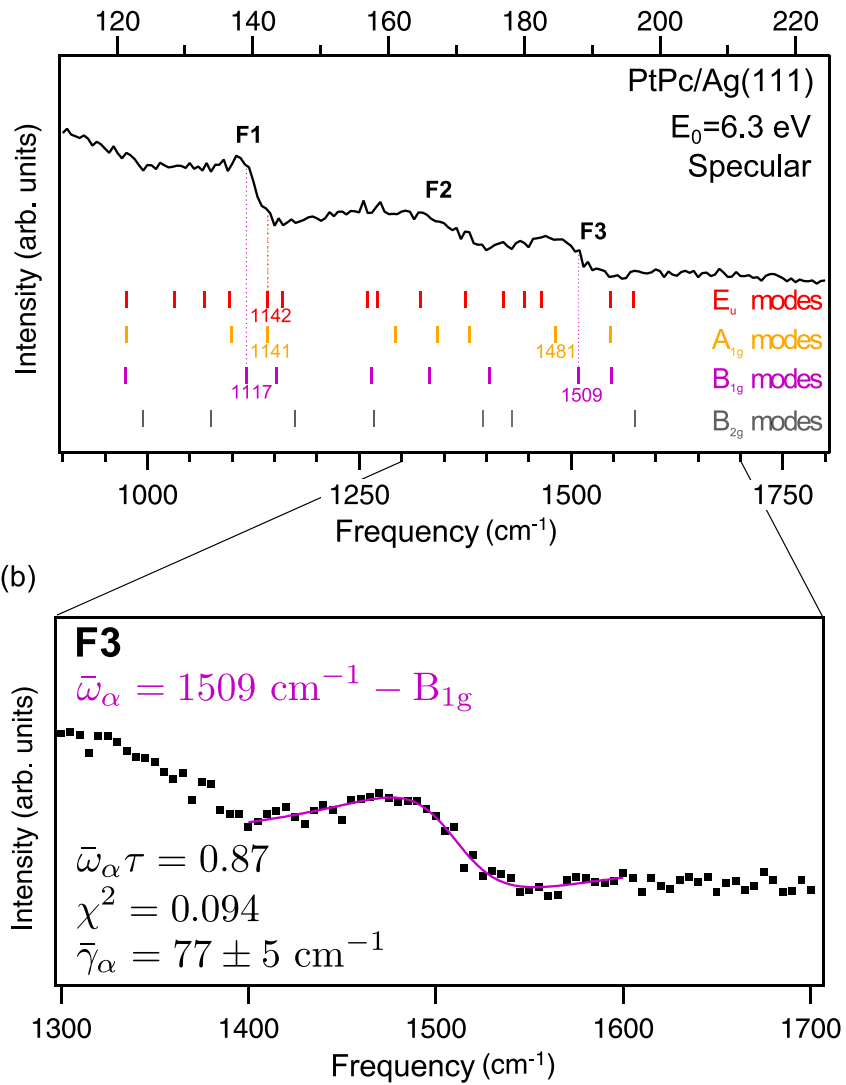

FIG. 3. (a) Zoom of the specular HREELS spectrum of the ordered monolayer phase spectrum of PtPc on $\operatorname{Ag}(111)$ showing the asymmetric peaks F1, F2, and F3 together with the calculated vibrational modes (vertical bars). Each peak has been fitted using all the calculated vibrations marked in panel (a), and all the $\bar{\omega}_{\alpha}$ values which give reasonable fits of F1, F2, and F3 are shown with dotted lines. (b) Best fit of $\mathbf{F 3}$ using $\bar{\omega}_{\alpha}=1509 \mathrm{~cm}^{-1}-B_{1 \mathrm{~g}}$.

of $\bar{\omega}_{\alpha}$ that are determined by DFT calculations of an isolated molecule. All the calculated $\bar{\omega}_{\alpha}$ frequencies in the range from 900 to $1800 \mathrm{~cm}^{-1}$ have been tested [Fig. 3(a)]. The fits can be found in the Supplemental Material [29]. For a given Fano peak, the best fits are selected first on the basis of the smallest $\chi^{2}$ value(s) within all possible $\alpha$ modes, and second the value of $\bar{\gamma}_{\alpha}$ must be below $100 \mathrm{~cm}^{-1}$ [50]. The few modes that yield reasonable fits for $\mathbf{F 1}$ are summarized in Table III. Because F1 may possibly be assigned to a mode with an $A_{1 \mathrm{~g}}$ irreducible representation in the $D_{4 h}$ point group that maps onto a mode belonging to a totally symmetric irreducible representation in any subgroup of $D_{4 h}$, it is not possible to identify the symmetry of the molecule-substrate complex by considering this mode. As clearly seen in Fig. 3(a), F2 appears much broader than F1 and F3. This is confirmed by the fits that show $\bar{\gamma}_{\alpha}$ values always larger than $125 \mathrm{~cm}^{-1}$ [29]. This is an indication that several vibrational modes contribute to $\mathbf{F 2}$, and therefore renders an accurate assignment of this mode impossible.

We now turn to $\mathbf{F 3}$ at about $1500 \mathrm{~cm}^{-1}$. Using calculated frequencies in the fit, by far the best fit is achieved with the $B_{1 \mathrm{~g}}$ mode at $\bar{\omega}_{\alpha}=1509 \mathrm{~cm}^{-1}$; see Fig. 3(b). The fitted value of $\bar{\gamma}_{\alpha}$ $\left(77 \pm 5 \mathrm{~cm}^{-1}\right)$ compares well to the results for $\mathrm{ZnPc} / \mathrm{Ag}(110)$ $\left(60 \mathrm{~cm}^{-1}\right)$ [34]. We stress again that $\bar{\omega}_{\alpha}$ is not a fitting 
parameter. If a strong renormalization $\omega_{\alpha} \rightarrow \bar{\omega}_{\alpha}$ is expected, then also the $A_{1 \mathrm{~g}}$ mode at $1546 \mathrm{~cm}^{-1}$ could be a candidate and the assignment remains ambiguous. However, on the basis of the overall agreement between DFT calculation and experimental frequencies as documented in Table I we believe that the correct assignment is the $B_{1 \mathrm{~g}}$ mode at $1509 \mathrm{~cm}^{-1}$. According to the correlation table in Table II, the $B_{1 \mathrm{~g}}$ mode maps to a totally symmetric $A_{1}$ mode for the $C_{2 v}\left(\sigma_{v}\right)$ subgroup, to the totally symmetric $A^{\prime}$ for the $C_{s}\left(\sigma_{v}\right)$ subgroup, or to the totally symmetric $A$ mode for the $C_{2}$ subgroup. Hence, the symmetry of the molecule substrate complex must be $C_{2 v}\left(\sigma_{v}\right)$, $C_{s}\left(\sigma_{v}\right)$, or $C_{2}$.

However, the mere appearance of the $B_{1 \mathrm{~g}}$ mode in the spectrum does not yet clarify the origin of the symmetry reduction $D_{4 h} \rightarrow C_{2 v}\left(\sigma_{v}\right), C_{s}\left(\sigma_{v}\right)$, or $C_{2}$. In principle, it is possible that because of a geometric distortion of the molecule $\mu_{\alpha}^{\text {ion }}$ is tilted out of the surface plane and acquires a perpendicular component. But in this case $\mu_{\alpha}$ would necessarily remain real, and we would observe a conventional, symmetric line shape for F3. This is evidently not the case. Its Fano line shape shows that $\mu_{\alpha}$ of $\mathbf{F 3}$ is complex. Hence, there must be a phase-shifted electronic contribution $\mu_{\alpha}^{\mathrm{el}}$ to its overall dynamical dipole moment $\mu_{\alpha}$.

We have already mentioned above that for PtPc/Ag(111) the LUMO is the most likely partner in IDCT, because it becomes filled upon adsorption and straddles the Fermi energy. Note that the reduction of the point group of the molecule-substrate complex from $D_{4 h}$ implies a lifting of the degeneracy of the LUMO. For example, in the case of the $C_{2 v}\left(\sigma_{v}\right)$ point group the two degenerate orbitals of the $E_{\mathrm{g}}$ representation map to two nondegenerate orbitals with a $B_{1}$ and $B_{2}$ irreducible representation. Moreover, a comparison of the elongation pattern of F3, which predominantly involves the stretching of the $\mathrm{C}-\mathrm{N}$ bonds in the porphyrazine macrocycle [Fig. 2(c)], with the lobular structure of the LUMO [Fig. 2(b)] shows that the ionic motion of $\mathbf{F 3}$ should indeed couple well to the LUMO. Specifically, the stretching of the $\mathrm{C}-\mathrm{N}$ bonds modulates the electron density along these bonds. Since the LUMO has bonding character with respect to them, the modulation of the electron density will have a strong impact on the energy of the LUMO. We note that it is the charge density along critical bonds, i.e., the square of the wave function irrespective of its phase, that determines the coupling to the orbital in question. In fact, this is the underlying physical reason why in IDCT orbitals transforming to any arbitrary one-dimensional representation may couple to any totally symmetric vibrational mode, as we have shown above on the basis of a general group-theory argument: Irrespective of the representation, the square of the wave function is totally symmetric. For this reason, the $B_{1}$ and $B_{2}$ orbitals of Fig. 2(b) may in fact couple to the F3 mode in Fig. 2(c). Comparing Figs. 2(b) and 2(c) moreover shows that exciting $\mathbf{F 3}$ with positive amplitude has the same effect on the $B_{1}$-LUMO as exciting $\mathbf{F 3}$ with negative amplitude has on the $B_{2}$-LUMO. Hence, if $B_{1}$ and $B_{2}$ were still degenerate, dynamic filling and depletion of the two would be $180^{\circ}$ out of phase (assuming that the phase lag relative to the ionic movement is the same for both LUMOs, which is expected to be a good approximation, in spite of the observed symmetry breaking; see below). In other words, in one half of the vibrational oscillation cycle the $B_{1}$-LUMO

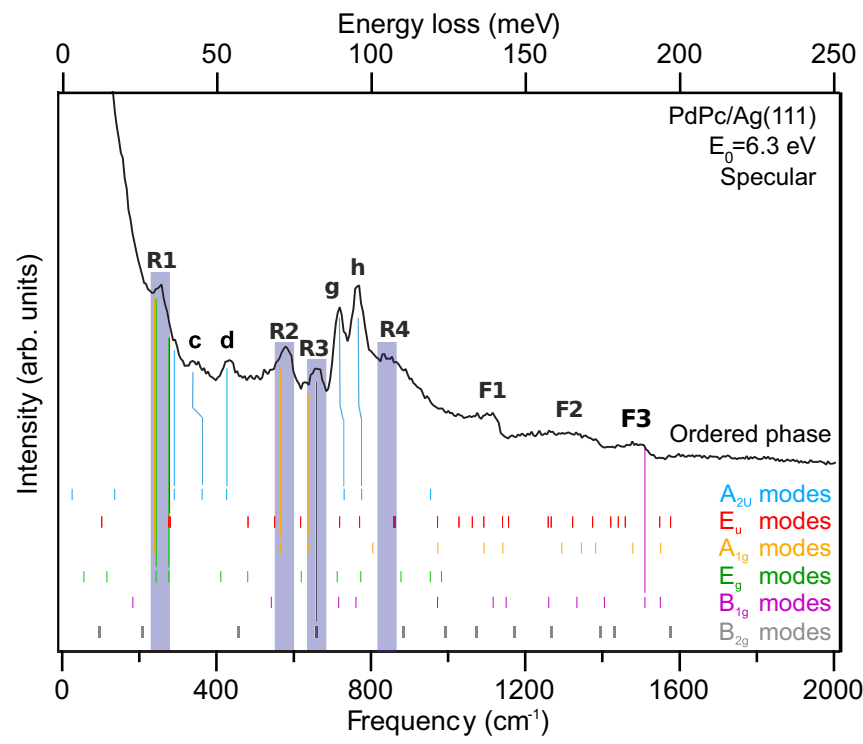

FIG. 4. Specular HREELS spectra of the PdPc ordered monolayer phase on $\mathrm{Ag}(111)$. The simulated vibrational modes of the isolated molecule are shown as thick colored vertical bars. The energy ranges used for assignment around the centroids of $\mathbf{R} \mathbf{1}$ to $\mathbf{R} \mathbf{4}$ are depicted as semitransparent blue boxes, and possible assignments are indicated by thin vertical bars (see text).

would be filled, while the $B_{2}$-LUMO would be depleted, and vice versa in the other half. Overall, no $\mu_{\alpha}^{\mathrm{el}}$ would prevail (small differences between the IDCT in the $B_{1}$ - and $B_{2}$-LUMOs notwithstanding).

However, in the experimental spectrum a substantial $\mu_{\alpha}^{\mathrm{el}}$ is observed, as argued above. This can mean two things. Either the two LUMOs are still degenerate and their dynamic couplings to the $\mathbf{F 3}$ vibration are different, or the LUMO levels $B_{1}$ and $B_{2}$ are indeed split. We consider it unlikely that the static level positions of the LUMOs are not affected by symmetry breaking, while their dynamic couplings to $\mathbf{F 3}$ are. Therefore, we conclude that the $B_{1}$ - and $B_{2}$-LUMO levels must be split; i.e., the degeneracy of the PtPc LUMO is lost upon adsorption on $\mathrm{Ag}(111)$. One may speculate that this LUMO splitting proceeds via a geometric distortion that is induced by the environment [the $\operatorname{Ag}(111)$ surface], enhanced by a Jahn-Tellerlike internal stabilization of this external geometric distortion via the splitting of the electronic LUMO state $[16,19,20]$.

\section{PALLADIUM-PHTHALOCYANINE ON Ag(111)}

PdPc molecules adsorbed on $\mathrm{Ag}(111)$ show similar longrange order to that of PtPc/Ag(111). A two-dimensional gas phase is found after annealing the thick layer up to $730 \mathrm{~K}$ and an ordered phase is detected in LEED for lower annealing temperatures, that is at higher coverages.

In Fig. 4 the specular HREELS spectrum of the PdPc ordered monolayer phase is shown together with the simulated results for the isolated molecule. The assignment of the experimental vibrational frequencies can be found in the Supplemental Material [29]. As in the case of PtPc, the in-plane $E_{\mathrm{u}}$ modes are absent and only the dipole scattering allowed out-of-plane $A_{2 \mathrm{u}}$ modes of the isolated molecule are present. 
TABLE IV. Possible assignments of the $\mathbf{R}$ and $\mathbf{F}$ modes observed in the ordered monolayer phase of PdPc on $\mathrm{Ag}(111)$. The $\mathbf{F}$ mode assignments are based on the fit results.

\begin{tabular}{lc}
\hline \hline Mode & Symmetry \\
\hline R1 & $A_{1 \mathrm{~g}} / E_{\mathrm{g}} / E_{\mathrm{u}}(x, y)$ \\
$\mathbf{R 2}$ & $A_{1 \mathrm{~g}}$ \\
$\mathbf{R 3}$ & $A_{1 \mathrm{~g}} / B_{2 \mathrm{~g}}$ \\
$\mathbf{F 1}$ & $A_{1 \mathrm{~g}} / E_{\mathrm{u}}(x, y) / B_{1 \mathrm{~g}}$ \\
$\mathbf{F 3}$ & $B_{1 \mathrm{~g}}$ \\
\hline \hline
\end{tabular}

This indicates an essentially flat adsorption geometry. The c, $\mathbf{g}$, and $\mathbf{h}$ modes are shifted to lower frequencies by approximately 37,18 , and $5 \mathrm{~cm}^{-1}$ with respect to theory, respectively. The larger shift of the c mode for PdPc $\left(37 \mathrm{~cm}^{-1}\right)$ than for PtPc $\left(32 \mathrm{~cm}^{-1}\right)$ suggests a marginally stronger interaction of PdPc with the $\operatorname{Ag}(111)$ substrate.

The presence of $\mathrm{R}$ modes in Fig. 4 suggests a reduction of the molecular symmetry upon adsorption. In addition to the $\mathbf{R 1}, \mathbf{R 2}$, and $\mathbf{R 3}$ peaks that are also observed for PtPc/Ag(111), the PdPc spectrum shows a peak at $850 \mathrm{~cm}^{-1}$, labeled R4. Due to the large width of $\mathbf{R 4}$, an assignment is not possible. Likely, several components participate in it. Possible assignments of $\mathbf{R} 1, \mathbf{R} 2$, and $\mathbf{R 3}$ are listed in Table IV using a range of $50 \mathrm{~cm}^{-1}$ around their centroid. Since there is the possibility to assign the $\mathbf{R 1}, \mathbf{R 2}$, and $\mathbf{R 3}$ peaks to modes with a $A_{1 \mathrm{~g}}$ irreducible representation, they would then be detectable in HREELS in specular geometry irrespective of the point group of the molecule-substrate complex $\left(C_{4 v}, C_{2 v}, C_{s}, C_{4}\right.$, or $\left.C_{2}\right)$; cf. Table II.

Three asymmetric peaks (F1, F2, and F3) are observed between 1000 and $1600 \mathrm{~cm}^{-1}$, which indicates that several vibrational modes are involved in an IDCT. Using Eq. (19), we single out vibrational frequencies given by DFT calculations for isolated $\operatorname{PdPc}\left(\bar{\omega}_{\alpha}\right)$ that yield the best fit of the experimental data as explained in Sec. III C 3. As in the case of PtPc, several modes yield a reasonable fit for F1. Due to the large $\bar{\gamma}_{\alpha}$ value of F2, a clear assignment is not possible. F3 can be fitted only with $\bar{\omega}_{\alpha}=1506 \mathrm{~cm}^{-1}$, a mode which belongs to the $B_{1 \mathrm{~g}}$ irreducible representation of the $D_{4 h}$ point group (see Fig. 5), and a $B_{1 \mathrm{~g}}$ mode can only be detected in HREELS in the specular direction if the fourfold molecular symmetry is reduced to $C_{2 v}\left(\sigma_{v}\right), C_{s}\left(\sigma_{v}\right)$, or $C_{2}$ (see Table II). As in the case of $\mathrm{PtPc}$, this demonstrates that a preferential charge transfer

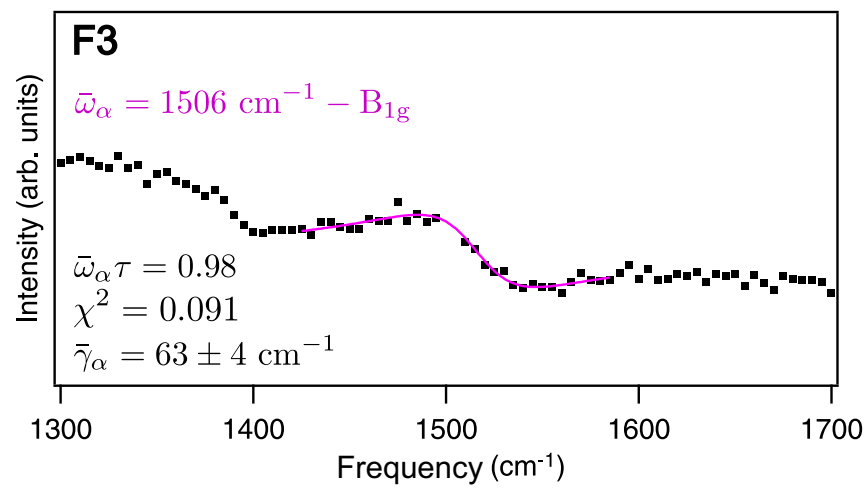

FIG. 5. Best fit of $\mathbf{F 3}$ using $\bar{\omega}_{\alpha}=1506 \mathrm{~cm}^{-1}-B_{1 \mathrm{~g}}$, for the ordered monolayer PdPc phase on $\operatorname{Ag}(111)$.

occurs in one of the two LUMOs. Thus, the $\operatorname{Ag}(111)$ substrate lifts the degeneracy of the LUMO also in the case of PdPc.

\section{SUMMARY AND CONCLUSION}

In conclusion, the vibrational properties of $\mathrm{Pt}$ - and $\mathrm{Pd}-$ phthalocyanine molecules on the $\mathrm{Ag}(111)$ surface show that both molecules adsorb with their molecular plane parallel to the surface in the ordered monolayer phase. The redshift of some of the out-of-plane modes reflects a moderate interaction between the meso-tetraazaporphin (porphyrazin) macrocycle of the molecule and the $\mathrm{Ag}$ substrate. The presence of Raman vibrational modes proves the lowering of the molecular symmetry from the $D_{4 h}$ group of the isolated molecule upon adsorption on $\operatorname{Ag}(111)$. The asymmetrical line shape of some of the molecular vibrational modes further demonstrates that charge transfer to the molecule is involved in the symmetry reduction to at least $C_{2 v}\left(\sigma_{v}\right)$ or lower. Therefore, this study shows that HREELS is a valuable tool to determine the origin of the molecular degeneracy lifting upon adsorption on a metal surface.

\section{ACKNOWLEDGMENTS}

F.C.B. acknowledges financial support from the Initiative and Networking Fund of the Helmholtz Association, Postdoc Programme VH-PD-025. J.S. thanks Prof. Dr. P. Jakob, Philipps Universität Marburg, and Dr. P. Amsalem, Humboldt Universität Berlin, for fruitful discussions.
[1] R. H. Friend, R. W. Gymer, A. B. Holmes, J. H. Burroughes, R. N. Marks, C. Taliani, D. D. C. Bradley, D. A. Dos Santos, J. L. Brédas, M. Lögdlund, and W. R. Salaneck, Electroluminescence in conjugated polymers, Nature (London) 397, 121 (1999).

[2] P. W. M. Blom, V. D. Mihailetchi, L. J. A. Koster, and D. E. Markov, Device physics of polymer: Fullerene bulk heterojunction solar cells, Adv. Mater. 19, 1551 (2007).

[3] S. Sanvito, Molecular spintronics, Chem. Soc. Rev. 40, 3336 (2011).

[4] J. M. Gottfried, Surface chemistry of porphyrins and phthalocyanines, Surf. Sci. Rep. 70, 259 (2015).
[5] J. Blochwitz, M. Pfeiffer, T. Fritz, and K. Leo, Low voltage organic light emitting diodes featuring doped phthalocyanine as hole transport material, Appl. Phys. Lett. 73, 729 (1998).

[6] B. Crone, A. Dodabalapur, Y.-Y. Lin, R. W. Filas, Z. Bao, A. LaDuca, R. Sarpeshkar, H. E. Katz, and W. Li, Large-scale complementary integrated circuits based on organic transistors, Nature (London) 403, 521 (2000).

[7] G. Horowitz, Organic field-effect transistors, Adv. Mater. 10, 365 (1998).

[8] J. C. Conboy, E. J. C. Olson, D. M. Adams, J. Kerimo, A. Zaban, B. A. Gregg, and P. F. Barbara, Impact of solvent vapor 
annealing on the morphology and photophysics of molecular semiconductor thin films, J. Phys. Chem. B 102, 4516 (1998).

[9] Y. Wang, J. Kröger, R. Berndt, and W. Hofer, Structural and electronic properties of ultrathin tin-phthalocyanine films on $\operatorname{Ag}(111)$ at the single-molecule level, Angew. Chem. Int. Ed. 48, 1261 (2009).

[10] S.-H. Chang, S. Kuck, J. Brede, L. Lichtenstein, G. Hoffmann, and R. Wiesendanger, Symmetry reduction of metal phthalocyanines on metals, Phys. Rev. B 78, 233409 (2008).

[11] H. Karacuban, M. Lange, J. Schaffert, O Weingart, Th. Wagner, and R. Möller, Substrate-induced symmetry reduction of CuPc on $\mathrm{Cu}(111)$ : An LT-STM study, Surf. Sci. 603, L39 (2009).

[12] Y. Wang, X. Ge, C. Manzano, J. Kröger, R. Berndt, W. A. Hofer, H. Tang, and J. Cerda, Supramolecular patterns controlled by electron interference and direct intermolecular interactions, J. Am. Chem. Soc. 131, 10400 (2009).

[13] R. Cuadrado, J. I. Cerdá, Y. Wang, G. Xin, R. Berndt, and H. Tang, CoPc adsorption on $\mathrm{Cu}(111)$ : Origin of the $\mathrm{C} 4$ to $\mathrm{C} 2$ symmetry reduction, J. Chem. Phys. 133, 154701 (2010).

[14] O. Snezhkova, J. Lüder, A. Wiengarten, S. R. Burema, F. Bischoff, Y. He, J. Rusz, J. Knudsen, M.-L. Bocquet, K. Seufert, J. V. Barth, W. Auwärter, B. Brena, and J. Schnadt, Nature of the bias-dependent symmetry reduction of iron phthalocyanine on $\mathrm{Cu}(111)$, Phys. Rev. B 92, 075428 (2015).

[15] N. F. Phelan and M. Orchin, Cross conjugation, J. Chem. Educ. 45, 633 (1968).

[16] C. Uhlmann, I. Swart, and J. Repp, Controlling the orbital sequence in individual $\mathrm{Cu}$-phthalocyanine molecules, Nano Lett. 13, 777 (2013).

[17] V. Feyer, M. Graus, P. Nigge, M. Wießner, R. G. Acres, C. Wiemann, C. M. Schneider, A. Schöll, and F. Reinert, Adsorption geometry and electronic structure of iron phthalocyanine on Ag surfaces: A LEED and photoelectron momentum mapping study, Surf. Sci. 621, 64 (2014).

[18] T. Niu, M. Zhou, J. Zhang, Y. Feng, and W. Chen, Dipole orientation dependent symmetry reduction of chloroaluminum phthalocyanine on $\mathrm{Cu}(111)$, J. Phys. Chem. C 117, 1013 (2013).

[19] H. A. Jahn and E. Teller, Stability of polyatomic molecules in degenerate electronic states. I. Orbital degeneracy, Proc. R. Soc. A 161, 220 (1937).

[20] H. Köpper, D. R. Yarkony, and H. Berentzen, The Jahn-Taller Effect: Fundamentals and Implications for Physics and Chemistry, Springer Series in Chemical Physics No. 97 (Springer, Berlin, 2009).

[21] P. Borghetti, A. El-Sayed, E. Goiri, C. Rogero, J. Lobo-Checa, L. Floreano, J. E. Ortega, and D. G. de Oteyza, Spectroscopic fingerprints of work-function-controlled phthalocyanine charging on metal surfaces, ACS Nano 8, 12786 (2014).

[22] P. Puschnig, S. Berkebile, A. J. Fleming, G. Koller, K. Emtsev, T. Seyller, J. D. Riley, C. Ambrosch-Draxl, F. P. Netzer, and M. G. Ramsey, Reconstruction of molecular orbital densities from photoemission data, Science 326, 702 (2009).

[23] P. Puschnig, E.-M. Reinisch, T. Ules, G. Koller, S. Soubatch, M. Ostler, L. Romaner, F. S. Tautz, C. Ambrosch-Draxl, and M. G. Ramsey, Orbital tomography: Deconvoluting photoemission spectra of organic molecules, Phys. Rev. B 84, 235427 (2011).

[24] K. Schönauer, S. Weiss, V. Feyer, D. Lüftner, B. Stadtmüller, D. Schwarz, T. Sueyoshi, C. Kumpf, P. Puschnig, M. G. Ramsey, F. S. Tautz, and S. Soubatch, Charge transfer and symmetry reduction at the $\mathrm{CuPc} / \mathrm{Ag}(110)$ interface studied by photoemission tomography, Phys. Rev. B 94, 205144 (2016).

[25] H. Ibach and D. L. Mills, Electron Energy Loss Spectroscopy and Surface Vibrations (Academic Press, New York, 1982).

[26] M. J. Frisch, G. W. Trucks, H. B. Schlegel, G. E. Scuseria, M. A. Robb, J. R. Cheeseman, G. Scalmani, V. Barone, B. Mennucci, G. A. Petersson, H. Nakatsuji, M. Caricato, X. Li, H. P. Hratchian, A. F. Izmaylov, J. Bloino, G. Zheng, J. L. Sonnenberg, M. Hada, M. Ehara, K. Toyota, R. Fukuda, J. Hasegawa, M. Ishida, T. Nakajima, Y. Honda, O. Kitao, H. Nakai, T. Vreven, J. A. Montgomery, Jr., J. E. Peralta, F. Ogliaro, M. Bearpark, J. J. Heyd, E. Brothers, K. N. Kudin, V. N. Staroverov, R. Kobayashi, J. Normand, K. Raghavachari, A. Rendell, J. C. Burant, S. S. Iyengar, J. Tomasi, M. Cossi, N. Rega, J. M. Millam, M. Klene, J. E. Knox, J. B. Cross, V. Bakken, C. Adamo, J. Jaramillo, R. Gomperts, R. E. Stratmann, O. Yazyev, A. J. Austin, R. Cammi, C. Pomelli, J. W. Ochterski, R. L. Martin, K. Morokuma, V. G. Zakrzewski, G. A. Voth, P. Salvador, J. J. Dannenberg, S. Dapprich, A. D. Daniels, Ó. Farkas, J. B. Foresman, J. V. Ortiz, J. Cioslowski, and D. J. Fox, Gaussian09 Revision D.01 (Gaussian Inc., Wallingford, CT, 2009).

[27] Z. Liu, X. Zhang, Y. Zhang, and J. Jiang, Theoretical investigation of the molecular, electronic structures and vibrational spectra of a series of first transition metal phthalocyanines, Spectrochim. Acta. 67A, 1232 (2007).

[28] V. G. Maslov, Interpretation of the electronic spectra of phthalocyanines with transition metals from quantum-chemical calculations by the density functional method, Opt. Spectrosc. 101, 853 (2006).

[29] See Supplemental Material at http://link.aps.org/supplemental/ 10.1103/PhysRevB.96.165410 for the LEED patterns, a detailed description of the $A_{2 \mathrm{u}}$ modes, all fits and a list of vibrational modes for PdPc on $\mathrm{Ag}(111)$.

[30] I. Kröger, B. Stadtmüller, C. Stadler, J. Ziroff, M. Kochler, A. Stahl, F. Pollinger, T.-L. Lee, J. Zegenhagen, F. Reinert, and C. Kumpf, Submonolayer growth of copper-phthalocyanine on Ag(111), New J. Phys. 12, 083038 (2010).

[31] B. Stadtmüller, I. Kröger, F. Reinert, and C. Kumpf, Submonolayer growth of CuPc on noble metal surfaces, Phys. Rev. B 83, 085416 (2011).

[32] C. Stadler, S. Hansen, I. Kröger, C. Kumpf, and E. Umbach, Tuning intermolecular interaction in long-range-ordered submonolayer organic films, Nat. Phys. 5, 153 (2009).

[33] R. L. Carter, Molecular Symmetry and Group Theory (John Wiley \& Sons, Inc., 1998).

[34] P. Amsalem, L. Giovanelli, J.-M. Themlin, and T. Angot, Electronic and vibrational properties at the $\mathrm{ZnPc} / \mathrm{Ag}(110)$ interface, Phys. Rev. B 79, 235426 (2009).

[35] W. Dou, Y. Tang, C. S. Lee, S. N. Bao, and S. T. Lee, Investigation on the orderly growth of thick zinc phthalocyanine films on $\operatorname{Ag}(100)$ surface, J. Chem. Phys. 133, 144704 (2010).

[36] W. Dou, S. Huang, R. Q. Zhang, and C. S. Lee, Molecule substrate interaction channels of metal-phthalocyanines on graphene on $\mathrm{Ni}(111)$ surface, J. Chem. Phys. 134, 094705 (2011).

[37] N. Ohta, R. Arafune, N. Tsukahara, N. Takagi, and M. Kawai, Adsorbed states of iron(II) phthalocyanine on $\mathrm{Ag}(111)$ studied 
by high-resolution electron energy loss spectroscopy, Surf. Interface Anal. 46, 1253 (2014).

[38] J. D. Baran and J. A. Larsson, Theoretical insights into adsorption of cobalt phthalocyanine on $\operatorname{Ag}(111)$ : A combination of chemical and van der Waals bonding, J. Phys. Chem. C 117, 23887 (2013).

[39] R. C. Powell, Symmetry, Group Theory, and the Physical Properties of Crystals, Lecture Notes in Physics, No. 824 (Springer, New York, 2010).

[40] Y. J. Chabal, Electronic Damping of Hydrogen Vibration on the W(100) Surface, Phys. Rev. Lett. 55, 845 (1985).

[41] J. Kröger, S. Lehwald, and H. Ibach, EELS study of the clean and hydrogen-covered Mo(110) surface, Phys. Rev. B 55, 10895 (1997).

[42] F. S. Tautz, M. Eremtchenko, J. A. Schaefer, M. Sokolowski, V. Shklover, and E. Umbach, Strong electron-phonon coupling at a metal/organic interface: PTCDA/Ag(111), Phys. Rev. B 65, 125405 (2002).

[43] I. Kröger, Adsorption of phthalocyanines on noble metal surfaces, Ph.D. thesis, Universität Würzburg, Germany, 2010.
[44] C. R. Braatz, G. Öhl, and P. Jakob, Vibrational properties of the compressed and the relaxed 1,4,5,8-naphthalene-tetracarboxylic dianhydride monolayer on $\operatorname{Ag}(111)$, J. Chem. Phys. 136, 134706 (2012).

[45] P. Rosenow, P. Jakob, and R. Tonner, Electron-vibron coupling at metal-organic interfaces from theory and experiment, J. Phys. Chem. Lett. 7, 1422 (2016).

[46] F. A. Cotton, Chemical Applications of Group Theory, 3rd ed. (John Wiley \& Sons, Inc., 1990).

[47] D. C. Langreth, Energy Transfer at Surfaces: Asymmetric Line Shapes and the Electron-Hole-Pair Mechanism, Phys. Rev. Lett. 54, 126 (1985).

[48] Ž. Crljen and D. C. Langreth, Asymmetric line shapes and the electron-hole pair mechanism for adsorbed molecules on surfaces, Phys. Rev. B 35, 4224 (1987).

[49] J. Kröger, Electron-phonon coupling at metal surfaces, Rep. Prog. Phys. 69, 899 (2006).

[50] $\bar{\gamma}_{\alpha}=60 \mathrm{~cm}^{-1}$ has been reported for a similar ( $\left.\mathrm{ZnPc}\right)$ molecule on $\operatorname{Ag}(110)$ in Ref. [34]. Therefore, we expect $\bar{\gamma}_{\alpha}$ values for PtPc and $\mathrm{PdPc}$ on $\mathrm{Ag}(111)$ to be close to $60 \mathrm{~cm}^{-1}$. 